\title{
BMJ Open Comparison of health-related quality of life in patients with atrial fibrillation treated with catheter ablation or antiarrhythmic drug therapy: a systematic review and meta- analysis protocol
}

\author{
Katherine S Allan, ${ }^{1}$ Shaunattonie Henry, ${ }^{1}$ Theresa Aves, ${ }^{2}$ Laura Banfield, ${ }^{3}$ \\ J Charles Victor, ${ }^{4}$ Paul Dorian, ${ }^{2,5,6}$ Jeff S Healey, ${ }^{7}$ Jason Andrade, ${ }^{8,9}$ \\ Sandra Carroll, ${ }^{1,7}$ Michael McGillion ${ }^{1,7}$
}

To cite: Allan KS, Henry S, Aves T, et al. Comparison of health-related quality of life in patients with atrial fibrillation treated with catheter ablation or antiarrhythmic drug therapy: a systematic review and metaanalysis protocol. BMJ Open 2017;7:e017577. doi:10.1136/ bmjopen-2017-017577

- Prepublication history and additional material for this paper are available online. To view please visit the journal (http:// dx.doi.org/10.1136/bmjopen2017-017577).

Received 2 May 2017 Revised 17 July 2017 Accepted 19 July 2017

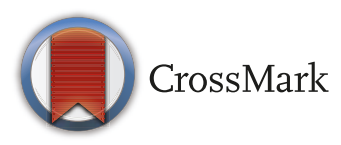

For numbered affiliations see end of article.

Correspondence to Dr Katherine S Allan; allank@mcmaster.ca, allank@ smh.ca

\section{ABSTRACT}

Introduction Atrial fibrillation (AF) is the most prevalent cardiac arrhythmia and causes patients considerable burden; symptoms such as palpitations and dyspnoea are common, leading to frequent emergency room visits. Patients with AF report reduced health-related quality of life (HQOL) compared with the general population; thus, treatments focus on the restoration of sinus rhythm to improve symptoms. Catheter ablation (CA) is a primary treatment strategy to treat AF-related burden in select patient populations; however, repeat procedures are often needed, there is a risk of major complications and the procedure is quite costly in comparison to medical therapy. As the outcomes after $\mathrm{CA}$ are mixed, an updated review that synthesises the available literature, on outcomes that matter to patients, is needed so that patients and their healthcare providers can make quality treatment decisions. The purpose of this review protocol is to extend previous findings by systematically analysing randomised controlled trials (RCTs) of CA in patients with AF and using metaanalytic techniques to identify the benefits and risks of $\mathrm{CA}$ with respect to HQOL and AF-related symptoms.

Methods and analysis We will include all RCTs that compare CA with antiarrhythmic drugs, or radiofrequency $\mathrm{CA}$ with cryoballoon $\mathrm{CA}$, in patients with paroxysmal or persistent AF. To locate studies we will perform comprehensive electronic database searches from database inception to 4 April 2017, with no language restrictions. We will conduct a quantitative synthesis of the effect of CA on HQOL as well as AF-related symptoms and the number of CA procedures needed for success, using meta-analytic techniques.

Ethics and dissemination No ethical issues are foreseen and ethical approval is not required given that this is a protocol. The findings of the study will be reported at national and international conferences, and in a peerreviewed journal using the Preferred Reporting Items for Systematic Reviews and Meta-Analyses (PRISMA) guidelines.
Strengths and limitations of this study

This systematic review and meta-analysis is the first to focus on patient-oriented treatment outcomes, including health-related quality of life (HQOL) and atrial fibrillation (AF)-related symptoms.

- This study will identify the benefits and risks of catheter ablation using differing energy sources compared with antiarrhythmic drugs, with respect to HQOL, AF-related symptoms and the number of repeat procedures needed for success.

- This systematic review and meta-analysis only focuses on randomised controlled trials and does not consider evidence from observational studies.

Trial registration number In accordance with the guidelines, our systematic review protocol was registered with the International Prospective Register of Systematic Reviews (PROSPERO) on 6 March 2017 and was last updated on 6 March 2017 (registration number CRD42017057427).

Protocol amendments Any protocol amendments will be documented on the International Prospective Register of Systematic Reviews (PROSPERO) and in the final manuscript and indicated as such.

\section{INTRODUCTION}

\section{Description of the condition}

Atrial fibrillation (AF) is the most common type of arrhythmia, affecting an estimated 33.5 million individuals globally. ${ }^{1}$ This arrhythmia causes rapid, irregular and chaotic electrical activity in the atria, often causing symptoms of palpitations, shortness of breath, effort intolerance or fatigue. ${ }^{2}$ Patients with $\mathrm{AF}$ are usually classified according to the duration of their AF episodes: AF is called 
paroxysmal when termination is spontaneous or it is resolved with intervention within 7 days; persistent $\mathrm{AF}$ is where patients remain in $\mathrm{AF}$ for greater than 7 days' duration; and permanent $\mathrm{AF}$ is when both the patient and physician choose to accept the $\mathrm{AF}$ and not pursue further attempts at sinus rhythm restoration. ${ }^{3}$

The societal burden of AF is considerable; compared with healthy controls, the general population and patients with coronary artery disease, patients with $\mathrm{AF}$ consistently report lower scores across health-related quality of life (HQOL) dimensions. ${ }^{2}{ }^{4-8}$ The Canadian Trial of Atrial Fibrillation, a randomised controlled trial (RCT) of low-dose amiodarone versus conventional antiarrhythmic drugs (AADs), observed that women reported significantly poorer physical and functional health; however, mental health and well-being scores were virtually identical for both sexes. ${ }^{9}$ Furthermore, symptoms such as palpitations, fatigue and dyspnoea are common, leading to frequent emergency room visits at an approximate cost of $\$ 49$ million annually. ${ }^{10}$

Management of patients with AF includes two objectives: the use of anticoagulation therapy to reduce the risk of stroke, as well as medications that control either the rate or the rhythm to alleviate symptoms. According to the latest guidelines, ${ }^{11} 12$ management of $\mathrm{AF}$ is centred on symptomatic improvement, as well as reduction in morbidity and mortality. While ventricular rate control can be very effective in some individuals, a significant proportion of patients remain symptomatic despite optimal rate control. ${ }^{13}$ For these, restoration and maintenance of sinus rhythm with AADs can alleviate symptoms and improve exercise capacity/quality of life. ${ }^{12}$ Unfortunately these medications have only modest efficacy at maintaining sinus rhythm over the long term ${ }^{14}$ and are associated with serious side effects ${ }^{15}$; this can limit their long-term use, especially in younger patients. Neither rate or rhythm control strategies have been shown to demonstrate superiority with respect to mortality nor reducing stroke risk. ${ }^{67}$ Thus, other factors such as patient preference or improved HQOL should help guide treatment options.

\section{Description of the intervention and therapeutic benefit}

Catheter ablation (CA) is a common strategy to control AF symptoms in select patient populations, such as highly symptomatic paroxysmal AF, or to control AF in cases where pharmacological therapies have failed or cannot be tolerated. ${ }^{11}$ The principal driver for choosing this treatment option is the improvement of AF-related symptoms and HQOL. Cumulative published evidence confirms the superiority of CA over AADs in reducing AF recurrence, ${ }^{14}{ }^{15}$ wherein patients often realise greater gains in HQOL compared with those treated with pharmacological therapy alone. ${ }^{16-18}$ For example, in three RCTs that compared CA with AAD therapy in patients with paroxysmal AF, CA was associated with significant improvement in baseline HQOL scores, as measured by the physical and mental component summary scores, of the SF-36 survey. ${ }^{1619}{ }^{20}$ These results were corroborated in the recent Medical Antiarrhythmic Treatment or Radiofrequency Ablation in Paroxysmal Atrial Fibrillation (MANTRA-PAF) trial, which showed that HQOL scores at 5 years remained significantly improved from baseline for patients within both the $\mathrm{AAD}$ and $\mathrm{CA}$ treatment groups; however, no between-group differences were observed across the SF-36 subscales. ${ }^{21}$

While the benefits of CA are clear, the procedure is not without drawbacks. Repeat procedures may be required to maintain sinus rhythm, even among those with paroxysmal $\mathrm{AF}$ and associated structural heart disease. ${ }^{22}$ For example, the documented success rate of $\mathrm{CA}$, in the absence of AAD therapy, is $60 \%-75 \%$ following one CA procedure. Success rates have been shown to increase to $75 \%-90 \%$ following a second procedure. ${ }^{23}$ In more complex patient populations, such as those with advanced structural heart disease, the success rates are lower. ${ }^{24} 25$ In a prospective multicentre study of 1400 patients with paroxysmal and persistent $\mathrm{AF}$ who underwent $\mathrm{CA}$, the success rates were $10 \%-15 \%$ lower than those described for paroxysmal AF; three-quarters of patients with both paroxysmal and persistent $\mathrm{AF}$ required repeat ablations to achieve freedom from arrhythmia recurrence, after which $92.4 \%$ of patients with paroxysmal $\mathrm{AF}$ and $84 \%$ of patients with persistent $\mathrm{AF}$ remained free of $\mathrm{AF}^{24}$ Lower success rates have also been reported in patients with $\mathrm{AF}$ with reduced ejection fractions due to structural heart disease. ${ }^{25}$ Moreover, CA confers an approximate risk of $5 \%$ for major procedural complications (eg, tamponade, stroke and so on), ${ }^{26}$ and is more costly than pharmacological therapy. ${ }^{28}$

Given that the evidence for $\mathrm{CA}$ as an effective treatment strategy for patients with AF is mixed, particularly for important patient outcomes such as HQOL and AF-related symptoms, it is vital to synthesise this literature, so that patients and their healthcare providers can make quality treatment decisions. As previous meta-analyses have focused primarily on the effectiveness of CA in preventing $\mathrm{AF}$ recurrence, there is an urgent need to perform an updated review, focusing on outcomes that have the most impact on patients.

\section{Objective}

The primary objective of this review is to assess the impact of CA therapy performed with either radiofrequency or cryoablation energy compared with AADs on HQOL in treating patients with either paroxysmal or persistent AF. The secondary objective of this review is to measure the frequency and severity of AF-related symptoms and the frequency of repeat procedures to maintain sinus rhythm.

\section{Research questions}

1. In patients with paroxysmal or persistent AF, does CA compared with AAD therapy improve HQOL?

2. In patients with paroxysmal or persistent AF, does CA compared with AAD therapy decrease the frequency and severity of AF-related symptoms? 
3. In patients with paroxysmal or persistent AF, how many CA procedures are needed to achieve freedom from $\mathrm{AF}$ recurrence?

\section{METHODS}

\section{Eligibility criteria}

Studies will be selected according to the criteria outlined below.

\section{Types of studies}

We will include RCTs that evaluate CA (any energy modality) compared with (1) AADs or (2) CA of a differing energy modality (eg, cryoablation vs radiofrequency energy), irrespective of blinding, publication status or language. Observational studies or RCTs that perform CA on other patient populations (ie, heart failure, coronary artery bypass grafting or valve and so on) will be excluded. Surgical ablation studies are beyond the scope of this review and will be excluded. CA for atrial flutter will also be excluded.

\section{Types of participants}

The participants of the study are male or female patients of any age or ethnicity who have been diagnosed with paroxysmal or persistent AF.

For the purposes of this review, we will include any patient with paroxysmal $\mathrm{AF}$ or persistent $\mathrm{AF}$ as defined by the study authors. Patients in permanent AF will be excluded from the analysis.

\section{Types of interventions}

CA is a procedure that targets and electrically isolates the pulmonary veins by delivering lesions immediately outside the ostia of the pulmonary veins or along a wider area in the left atrium encircling the veins. ${ }^{29}$ Additionally, operators can target and ablate non-pulmonary vein triggers or atrial areas thought to be responsible for maintaining $\mathrm{AF} .^{29}$ Only studies that use either radiofrequency energy or cryoablation energy will be included.

Any AAD (class I or III drugs) ${ }^{30}$ will be included as the comparator, as defined by the study authors.

\section{Types of outcome measures \\ Primary}

Patient important outcome measures will include HQOL, however it is measured by generic (eg, 36-Item Short Form Health Survey (SF-36), EuroQol five-dimension scale (EQ5D) and disease-specific (eg, Atrial Fibrillation Effect on QualiTy-of-life (AFEQT)) questionnaires. If data are disaggregated, HQOL will be compared based on the physical and mental component summaries and on the eight differing subscales of the SF-36 Survey.

\section{Secondary}

Secondary outcomes will include the frequency and severity of AF-related symptoms however they are measured, as well as the number of repeat procedures needed to maintain sinus rhythm.

\section{Search methods for identification of studies Electronic searches}

A comprehensive search strategy will be developed and carried out to identify published, in-press and unpublished studies, as well as ongoing trials. No language restrictions will be applied. The search will be conducted from database inception to 4 April 2017. The search strategy will be developed using a combination of keywords and database-specific subject headings for the following concepts: 'atrial fibrillation', 'catheter ablation' and 'randomised controlled trial'. Boolean operators 'OR' and 'AND' will be used to combine related terms and discrete concepts, respectively. The electronic search strategy for Medline is shown in the online supplementary appendix.

The search will include the following databases: Cochrane Central Register of Controlled Trials (CENTRAL), Cochrane Database of Systematic Reviews (CDSR), Medline, Cumulative Index to Nursing and Allied Health Literature (CINAHL) and Embase.

\section{Searching other resources}

We will examine the citations of included studies to identify additional studies not identified in the electronic search. Ongoing trials will be identified using the WHO International Clinical Trials Registry Platform and ClinicalTrials.gov. Investigators of trials in progress will be contacted if their completion date is reached. We will also contact experts to ask if they know of additional studies, including unpublished data.

\section{Identification and selection of studies}

Literature search results will be uploaded to Distiller Systematic Review (DSR) software. KSA will develop and test forms for level 1 and level 2 screening based on the inclusion and exclusion criteria. Citation abstracts will be uploaded with screening questions to DSR. Full-text articles will be screened on Reference Manager, with results captured in DSR. Prior to level 1 and level 2 screening, calibration exercises will be undertaken to pilot and refine the screening questions.

Three reviewers (KSA, TA, SH) will independently examine the titles and abstracts retrieved by the search. The full text of the abstracts will be reviewed and independently assessed for study inclusion/exclusion criteria. Studies will be included for full-text review on the basis of agreement between two reviewers or when there is disagreement or uncertainty. Three authors will then confirm which studies meet all inclusion criteria by independently reviewing the full text of potentially relevant articles. Data from multiple reports of the same study will be linked together and used to supplement information obtained from the primary report.

A summary of the study selection process will be presented using the PRISMA (Preferred Reporting Items for Systematic Reviews and Meta-analyses) flow chart. ${ }^{31}$ 


\section{Data extraction and management}

Using standardised forms in DSR, three reviewers (KSA, TA, SH) will independently perform data extraction in pairs, with discrepancies resolved through consensus. If consensus cannot be reached, a fourth reviewer (MM) will make a final judgement on the data. To ensure consistency across reviewers, we will conduct calibration exercises before starting abstraction. In the event of missing or unclear information, authors of the studies will be contacted for clarification. For reports published in languages other than English, collaborators from other countries will be invited to participate in screening, review and data extraction.

Data will be abstracted on the following variables:

- Study characteristics: including but not limited to location, duration of the trial, periods of data collection, follow-up duration, blanking periods, study design and number of trial sites

- Population characteristics: including but not limited to inclusion/exclusion criteria, number of participants randomised, number of participants analysed, attrition, and baseline characteristics such as age, sex, type of AF, prior AAD therapy and comorbidities

- Intervention characteristics: including but not limited to type of CA ablation, energy source, type of AAD, dosage and frequency

- Study outcome measures: including but not limited to HQOL, AF-related symptoms and number of repeat CA procedures.

Agreement between the three reviewers on study eligibility (Levels 1 and 2), data extraction and risk of bias assessment will be performed using the kappa statistic for inter-rater reliability. ${ }^{32}$

\section{Risk of bias assessment}

Risk of bias will be assessed as low risk, some risk of bias and high risk of bias using the following measures as outlined in the Cochrane Collaboration Risk of Bias 2.0 tool endorsed by Cochrane ${ }^{33}$ : adequacy of sequence generation, adequacy of allocation concealment, adequacy of blinding for participants, study personnel and outcome assessors, completeness of outcome data for each primary and secondary outcome, selective outcome reporting, and other potential sources of bias (ie, funding). Early stopping for benefit and observation of intention-to-treat principle will also be assessed. Risk of bias tables will be completed independently by the three reviewers (KSA, TA, SH) for all outcomes and compared for consensus.

Review Manager V.5.3.5 software will be used to enter and analyse the study data (RevMan 2014). GRADEpro GDT software will be used to generate a summary of findings and evidence profile tables (GRADEpro 2015). Confidence in effect estimates of the outcome measures will be rated according to the quality of evidence using the Grading of Recommendations Assessment, Development and Evaluation approach for systematic reviews. ${ }^{34}$ In this approach, RCTs will be ranked as high quality and downgraded for risk of bias, inconsistency, indirectness, imprecision and publication bias. ${ }^{34}$

Selective outcome reporting will be assessed as part of risk of bias. Publication bias will be evaluated using a funnel plot if 10 or more studies are included in the review. ${ }^{33}$

\section{Data synthesis}

Measures of treatment effect

When possible, results of included trials will be used in the meta-analysis. If the data are unavailable numerically, we will use approximations based on graphic output where possible. For studies reporting only means and IQRs, means and SD will be estimated. ${ }^{35}$ The weighted mean difference will be calculated for continuous outcomes measured on the same scale between studies, and the standardised mean difference will be calculated for continuous outcomes measured on different scales between studies. Point estimates and 95\% CIs will be reported. Studies presenting categorical HQOL or survival-based outcomes will be combined using a generic inverse variance method if common estimates of risk cannot be determined, as required. If data cannot be synthesised quantitatively, it will be summarised narratively.

\section{Dealing with missing data}

In the event of missing or unclear data, study authors will be contacted at the eligibility assessment and/or data abstraction stage. Secondary publications may be reviewed for missing data if they have the same study population.

\section{Assessment of heterogeneity}

Studies will be evaluated for clinical heterogeneity using the $\mathrm{X}^{2}$ test for homogeneity with an alpha=0.10 and the $\mathrm{I}^{2}$ statistic to quantify inconsistency. ${ }^{36} 37$

\section{Primary analysis}

Each outcome will be combined and calculated using RevMan V.5.3.5. If no significant heterogeneity exists, a fixed-effects model will be used to summarise effect sizes of primary and secondary outcomes. A random-effects model will be used if considerable unexplained heterogeneity exists between studies. Data from the included studies will be pooled at consistent time points across studies, 12 and 24 months post ablation to measure differences between treatments.

\section{Subgroup analyses}

Subgroup analyses to assess the causes of heterogeneity will be performed including the effect of CA versus AADs in treatment-naïve patients (eg, CA used as firstline treatment) versus after failed AADs (eg, CA is used as a secondary treatment following AADs), paroxysmal versus persistent $\mathrm{AF}$, men versus women, older (age >65) vs younger (age $<65$ ), and CA modality (radiofrequency vs cryoablation). 
We will also perform subgroup analyses based on questionnaire type, that is, either generic or disease-specific quality of life, to investigate if there are differing effects.

\section{Sensitivity analyses}

Sensitivity analyses will be planned to evaluate potential sources of bias resulting from variability in studies, such as risk of bias (ie, re-examine the study data omitting studies at high risk of bias).

\section{Author affiliations}

${ }^{1}$ School of Nursing, McMaster University, Hamilton, Ontario, Canada

${ }^{2}$ Division of Cardiology, St. Michael's Hospital, Toronto, Ontario, Canada

${ }^{3}$ Health Sciences Library, McMaster University, Hamilton, Ontario, Canada

${ }^{4}$ Institute of Health Policy, Management and Evaluation, University of Toronto, Toronto, Ontario, Canada

${ }^{5}$ Keenan Research Centre, Li Ka Shing Knowledge Institute, St. Michael's Hospital, Toronto, Ontario, Canada

${ }^{6}$ Department of Medicine, University of Toronto, Toronto, Ontario, Canada ${ }^{7}$ Population Health Research Institute, McMaster University, Hamilton, Ontario, Canada

${ }^{8}$ Department of Medicine, University of British Columbia, Vancouver, British Columbia, Canada

${ }^{9}$ Faculty of Health Sciences, Montreal Heart Institute, Université de Montréal, Montreal, Quebec, Canada

Contributors KSA is the guarantor. KSA, MM, SC, PD, JSH and JA were responsible for study concept and design. KSA, TA, SH, MM and SC drafted the manuscript. LB developed and performed the search strategy in conjunction with KSA. All authors contributed to the development of the selection criteria, the risk of bias assessment strategy and data extraction criteria. JCV provided statistical expertise. PD, JSH and JA provided content expertise. All authors provided feedback and approved the final manuscript.

Funding KSA was funded by an EB Eastburn Post-Doctoral Fellowship. This systematic review is funded by a Canadian Arrhythmia Network (CANet) CHAT Discovery Award. The study funder had no involvement in the preparation, conduct or writing of this protocol.

Competing interests JSH received grants from Medtronic and St Jude Medical.

Provenance and peer review Not commissioned; externally peer reviewed.

Open Access This is an Open Access article distributed in accordance with the Creative Commons Attribution Non Commercial (CC BY-NC 4.0) license, which permits others to distribute, remix, adapt, build upon this work non-commercially, and license their derivative works on different terms, provided the original work is properly cited and the use is non-commercial. See: http://creativecommons.org/ licenses/by-nc/4.0/

(c) Article author(s) (or their employer(s) unless otherwise stated in the text of the article) 2017. All rights reserved. No commercial use is permitted unless otherwise expressly granted.

\section{REFERENCES}

1. Chugh SS, Havmoeller R, Narayanan K, et al. Worldwide epidemiology of atrial fibrillation: a global burden of disease 2010 study. Circulation 2014;129:837-47.

2. Dorian P, Jung W, Newman D, et al. The impairment of healthrelated quality of life in patients with intermittent atrial fibrillation: implications for the assessment of investigational therapy. J Am Coll Cardiol 2000;36:1303-9.

3. Calkins H, Kuck KH, Cappato R, et al. 2012 HRS/EHRA/ECAS expert consensus statement on catheter and surgical ablation of atrial fibrillation: recommendations for patient selection, procedural techniques, patient management and follow-up, definitions, endpoints, and research trial design. Heart Rhythm 2012;9:632-96.

4. Hagens VE, Van Veldhuisen DJ, Kamp O, et al. Effect of rate and rhythm control on left ventricular function and cardiac dimensions in patients with persistent atrial fibrillation: results from the rate control versus electrical cardioversion for persistent atrial fibrillation (RACE) study. Heart Rhythm 2005;2:19-24.

5. Kang Y, Bahler R. Health-related quality of life in patients newly diagnosed with atrial fibrillation. Eur J Cardiovasc Nurs 2004;3:71-6.

6. Savelieva I, Paquette M, Dorian P, et al. Quality of life in patients with silent atrial fibrillation. Heart 2001;85:216-7.

7. Singh SN, Tang XC, Singh BN, et al. Quality of life and exercise performance in patients in sinus rhythm versus persistent atrial fibrillation: a veterans affairs cooperative studies program substudy. J Am Coll Cardiol 2006;48:721-30.

8. Thrall G, Lane D, Carroll D, et al. Quality of life in patients with atrial fibrillation: a systematic review. Am J Med 2006;119:448.e1-19.

9. Paquette M, Roy D, Talajic M, et al. Role of gender and personality on quality-of-life impairment in intermittent atrial fibrillation. $\mathrm{Am} \mathrm{J}$ Cardiol 2000;86:764-8.

10. O'Reilly DJ, Hopkins RB, Healey JS, et al. The burden of atrial fibrillation on the hospital sector in Canada. Can J Cardiol 2013;29:229-35.

11. Verma A, Cairns JA, Mitchell LB, et al. focused update of the canadian cardiovascular society guidelines for the management of atrial fibrillation. Can J Cardiol 2014;2014:1114-30.

12. Kirchhof P, Benussi S, Kotecha D, et al. ESC Guidelines for the management of atrial fibrillation developed in collaboration with EACTS. Eur Heart J 2016;2016:2893-962.

13. Kirchhof $\mathrm{P}, \mathrm{Ammentorp} \mathrm{B}$, Darius $\mathrm{H}$, et al. Management of atrial fibrillation in seven European countries after the publication of the 2010 ESC Guidelines on atrial fibrillation: primary results of the PREvention oF thromboemolic events--European registry in atrial fibrillation (PREFER in AF). Europace 2014;16:6-14.

14. Piccini JP, Lopes RD, Kong $\mathrm{MH}$, et al. Pulmonary vein isolation for the maintenance of sinus rhythm in patients with atrial fibrillation: a meta-analysis of randomized, controlled trials. Circ Arrhythm Electrophysiol 2009;2:626-33.

15. Shi LZ, Heng R, Liu SM, et al. Effect of catheter ablation versus antiarrhythmic drugs on atrial fibrillation: A meta-analysis of randomized controlled trials. Exp Ther Med 2015;10:816-22.

16. Jaïs $\mathrm{P}$, Cauchemez B, Macle L, et al. Catheter ablation versus antiarrhythmic drugs for atrial fibrillation: the A4 study. Circulation 2008;118:2498-505.

17. Reynolds MR, Walczak J, White SA, et al. Improvements in symptoms and quality of life in patients with paroxysmal atrial fibrillation treated with radiofrequency catheter ablation versus antiarrhythmic drugs. Circ Cardiovasc Qual Outcomes 2010;3:615-23.

18. Wazni OM, Marrouche NF, Martin DO, et al. Radiofrequency ablation vs antiarrhythmic drugs as first-line treatment of symptomatic atrial fibrillation: a randomized trial. JAMA 2005;293:2634-40.

19. Pappone C, Vicedomini G, Augello G, et al. Radiofrequency catheter ablation and antiarrhythmic drug therapy: a prospective, randomized, 4-year follow-up trial: the APAF study. Circ Arrhythm Electrophysiol 2011;4:808-14.

20. Wilber DJ, Pappone C, Neuzil P, et al. Comparison of antiarrhythmic drug therapy and radiofrequency catheter ablation in patients with paroxysmal atrial fibrillation: a randomized controlled trial. JAMA 2010;303:333-40.

21. Nielsen JC, Johannessen A, Raatikainen $P$, et al. Long-term efficacy of catheter ablation as first-line therapy for paroxysmal atrial fibrillation: 5-year outcome in a randomised clinical trial. Heart 2017;103:368-76.

22. Verma A, Natale A. Should atrial fibrillation ablation be considered first-line therapy for some patients? Why atrial fibrillation ablation should be considered first-line therapy for some patients. Circulation 2005;112:1214-22.

23. Finta B, Haines DE. Catheter ablation therapy for atrial fibrillation. Cardiol Clin 2004;22:127-45 ix.

24. Bhargava M, Di Biase L, Mohanty P, et al. Impact of type of atrial fibrillation and repeat catheter ablation on long-term freedom from atrial fibrillation: results from a multicenter study. Heart Rhythm 2009;6:1403-12.

25. Chen MS, Marrouche NF, Khaykin Y, et al. Pulmonary vein isolation for the treatment of atrial fibrillation in patients with impaired systolic function. J Am Coll Cardiol 2004;43:1004-9.

26. Gupta A, Perera T, Ganesan A, et al. Complications of catheter ablation of atrial fibrillation: a systematic review. Circ Arrhythm Electrophysiol 2013;6:1082-8.

27. Hoyt $\mathrm{H}$, Bhonsale $\mathrm{A}$, Chilukuri $\mathrm{K}$, et al. Complications arising from catheter ablation of atrial fibrillation: temporal trends and predictors. Heart Rhythm 2011;8:1869-74.

28. Blackhouse G, Assasi N, Xie F, et al. Cost-Effectiveness of Catheter Ablation for Rhythm Control of Atrial Fibrillation. Int J Vasc Med 2013;2013:1-11. 
29. Calkins H, Brugada J, Packer DL, et al. HRS/EHRA/ECAS expert consensus statement on catheter and surgical ablation of atrial fibrillation: recommendations for personnel, policy, procedures and follow-up. A report of the Heart Rhythm Society (HRS) Task Force on Catheter and Surgical Ablation of Atrial Fibrillation developed in partnership with the European Heart Rhythm Association (EHRA) and the European Cardiac Arrhythmia Society (ECAS); in collaboration with the American College of Cardiology (ACC), American Heart Association (AHA), and the Society of Thoracic Surgeons (STS). Endorsed and approved by the governing bodies of the American College of Cardiology, the American Heart Association, the European Cardiac Arrhythmia Society, the European Heart Rhythm Association, the Society of Thoracic Surgeons, and the Heart Rhythm Society. Europace 2007;9:335-79.

30. Gillis AM, Verma A, Talajic M, et al. Canadian Cardiovascular Society atrial fibrillation guidelines 2010: rate and rhythm management. Can J Cardiol 2011;27:47-59.
31. Moher D, Liberati A, Tetzlaff J, et al. Preferred reporting items for systematic reviews and meta-analyses: the PRISMA statement. PLoS Med 2009;6:e1000097.

32. Viera AJ, Garrett JM. Understanding interobserver agreement: the kappa statistic. Fam Med 2005;37:360-3.

33. Higgins JP, Altman DG, Gøtzsche PC, et al. The Cochrane Collaboration's tool for assessing risk of bias in randomised trials. BMJ 2011;343:d5928.

34. Guyatt GH, Oxman AD, Vist GE, et al. GRADE: an emerging consensus on rating quality of evidence and strength of recommendations. BMJ 2008;336:924-6.

35. Hozo SP, Djulbegovic B, Hozo I. Estimating the mean and variance from the median, range, and the size of a sample. BMC Med Res Methodol 2005;5:13

36. Higgins JP, Thompson SG. Quantifying heterogeneity in a metaanalysis. Stat Med 2002;21:1539-58.

37. Lau J, loannidis JP, Schmid CH. Quantitative synthesis in systematic reviews. Ann Intern Med 1997;127:820-6. 